\title{
Ejection Fraction Decreased, CTCAE
}

National Cancer Institute

\section{Source}

National Cancer Institute. Ejection Fraction Decreased, CT CAE. NCI Thesaurus. Code C143436.

The percentage computed when the amount of blood ejected during a ventricular contraction of the heart is compared to the amount that was present prior to the contraction. 